\title{
Linx
}

LinX Revue des linguistes de l'université Paris X Nanterre

45 | 2001

Invariants et variables dans les langues. Études typologiques

\section{Typologie et dynamiques des langues : les études acquisitionnelles sur les processus de morphologisation temporelle}

\section{Colette Noyau}

\section{OpenEdition}

\section{Journals}

Édition électronique

URL : http://journals.openedition.org/linx/841

DOI : 10.4000/linx.841

ISSN : 2118-9692

\section{Éditeur}

Presses universitaires de Paris Nanterre

Édition imprimée

Date de publication : 1 décembre 2001

Pagination : 177-184

ISSN : 0246-8743

\section{Référence électronique}

Colette Noyau, «Typologie et dynamiques des langues : les études acquisitionnelles sur les processus de morphologisation temporelle », Linx [En ligne], 45 | 2001, mis en ligne le 25 juin 2012, consulté le 19 avril 2019. URL : http://journals.openedition.org/linx/841; DOI : 10.4000/linx.841

Ce document a été généré automatiquement le 19 avril 2019

Département de Sciences du langage, Université Paris Ouest 


\title{
Typologie et dynamiques des langues : les études acquisitionnelles sur les processus de morphologisation temporelle
}

\author{
Colette Noyau
}

1 Depuis les années 80 , on a fait des progrès substantiels sur la description des dynamiques acquisitionnelles du langage, notamment sur l'acquisition de langues étrangères (ou secondes). Ces avancées ont été rendues possibles par les résultats d'études de grande envergure, longitudinales, c'est-à-dire suivant des locuteurs au long des étapes successives de construction d'une nouvelle langue, et translinguistiques, c'est-à-dire comparant les évolutions chez des groupes d'apprenants de différents couples de langues (langue première $=\mathrm{L} 1 /$ langue étrangère $=\mathrm{L} 2$ ), ce qui permet des généralisations importantes. Ces dynamiques peuvent être confrontées avec d'autres types de dynamiques du langage : celles de l'évolution diachronique, de la pidginisation et de la créolisation, des contacts de langues. La typologie linguistique est par ailleurs concernée par les phénomènes acquisitionnels enfantins, notamment autour de la théorie de la naturalité, par la voie de la recherche des conditions d'optimalité de l'émergence ou de la mise en œuvre des formes, et des invariants de complexification au cours de l'évolution diachronique, comme en témoigne entre autres le travail de Terzan-Kopecky (1997). L'acquisition de langues étrangères apporte de nouvelles pièces au dossier.

2 La dynamique de l'acquisition d'une langue étrangère (LE) peut être comprise en la considérant, plus que comme l'accroissement d'un répertoire linguistique, comme le fait de restructurations successives, et ces restructurations à leur tour peuvent être envisagées comme des modifications successives du poids respectif de différents niveaux d'organisation linguistique - de différents types d'indices - dans le traitement du langage par le locuteur apprenant. C'est l'idée directrice du modèle de la compétition, proposé par Bates \& Mc Whinney 1987, Mc Whinney \& Bates 1989, et développé en France par Kail 1990, 1995, 1997, qui permet de traiter de façon unifiée production, compréhension, 
acquisition, et d'intégrer la variation à la grammaire. Ces indices interviennent de façon différenciée selon les langues, et les travaux effectués dans cette perspective montrent notamment comment lors de l'acquisition du langage, les jeunes enfants en viennent petit à petit à accorder aux indices de leur langue le poids qu'ils ont dans la langue des adultes.

3 Les étapes élémentaires de l'acquisition d'une L2 sont désormais bien décrites, et on a identifié un palier potentiel de stabilisation lors de l'acquisition initiale (cf. Klein \& Perdue 1997, «basic variety») : le «lecte de base», constitué essentiellement d'un répertoire d'éléments lexicaux et d'un petit nombre de principes d'organisation des énoncés, de trois ordres : phrastique, sémantique, pragmatique, qui se retrouvent chez tous les apprenants, indépendamment des langues concernées, et ne comprennent pas de flexion verbale. Le poids relatif de ces trois types de principes évolue au cours de l'acquisition. Ils permettent au lecte de fonctionner relativement bien, mais entrent parfois en conflit, ce qui pousse à la retructuration du lecte.

4 Au-delà des étapes élémentaires, l'acquisition d'une L2 peut être vue en partie comme un processus de grammaticalisation. Non au sens initial restreint de Meillet (« attribution du caractère grammatical à un mot jadis autonome» (Meillet 1912, cité par Desclés \& Guentchéva 1997), mais au sens de Desclés \& Guentcheva (1997:28): «La grammaticalisation est un processus qui encode dans chaque langue, par des procédures formelles et régulières, c'est-à-dire par des marqueurs morpho-syntaxiques et phonosyntaxiques, un sous-système de significations constitutives d'une catégorie sémantique générale. »

5 Nous abordons ici la grammaticalisation acquisitionnelle d'un domaine linguistique, celui du temps en L2. La catégorie sémantique générale - ou notionnelle - se trouve selon les langues grammaticalisée de diverses façons, et met à contribution des phénomènes linguistiques très variés. Rappelons d'abord que la temporalité en général est exprimée par différentes gammes de moyens grammaticalisés et non grammaticalisés complémentaires, relevant de différents niveaux d'organisation du langage. Nous y incluons toute notion concernant a) les intervalles temporels associés aux situations et leurs relations entre eux et par rapport à des intervalles pris comme repères (relations temporelles), b) les perspectives temporelles adoptées par le locuteur (aspects), c) les caractéristiques temporelles inhérentes des procès (ce que certains auteurs persistent à appeler aspect (lexical ou inhérent).

\section{Le rôle des différents niveaux d'organisation du langage dans l'expression de la temporalité}

On pense d'abord à la temporalité inscrite dans la grammaire : morphologie flexionnelle - voire dérivationnelle (cf. les langues slaves), à laquelle s'ajoutent des schèmes composés V1-V2 ou phraséologiques plus ou moins fortement grammaticalisés. Mais la temporalité est exprimée également par : la temporalité inhérente des procès (diverses typologies sémantiques des prédications ont été proposées, cf. Gosselin \& François 1991), par des expressions lexicales et lexico-syntaxiques (connecteurs, adverbes et circonstants temporels), par la syntaxe des enchaînements de propositions (clause-combining), par l' organisation du discours (principe de l'ordre chronologique, configurations cadre temporel - événement inclus, etc.), et par des inférences fondées sur des savoirs contextuels ou des connaissances générales sur le monde. 
7 Les notions temporelles qui sont privilégiées dans une langue par le fait qu'elles sont grammaticalisées constituent des problèmes d'acquisition spécifiques, nous allons voir quelques exemples de la façon dont les apprenants s'y attaquent. Nous verrons que le développement de la temporalité en L2 peut être vu globalement comme un changement du poids des différents niveaux d'indices dans l'expression du domaine notionnel.

Un locuteur abordant une $\mathrm{L} E$ à la fois possède une expérience du temps des situations, du déroulement du temps, des relations temporelles - et une expérience via sa langue $d u$ temps dans le langage. C'est ce qu'il va lui falloir construire à nouveau dans une nouvelle langue. Et notamment il lui faudra découvrir quelles catégories de ce domaine sont privilégiées dans la L E par leur statut 'obstiné', obligatoire, marqué dans la morphologie. Double problème: identifier des marques, leur attribuer une valeur, le tout se jouant de façon infraconsciente, et non guidable par une intervention pédagogique descriptive même adéquate, comme on le verra.

On identifie des invariants dans les étapes de grammaticalisation temporelle à travers des couples de langues différents, en effectuant des généralisations inductives à partir d'études longitudinales translinguistiques (cf. Dietrich, Klein \& Noyau 1995). La grammaticalisation acquisitionnelle peut être confrontée avec ce qu'on sait des processus de grammaticalisation diachronique, lors de contacts de langues, ou dans la pidginisation / (dé-)créolisation. Elle peut donner lieu à des tentatives de grammaticalisation créative, mais elle se différencie de la grammaticalisation diachronique par le fait qu'il s'agit d'un processus de convergence vers la L C (Giacalone Ramat 1992).

10 Nous présentons ici une vue très synthétique des étapes de développement du temps et/ ou de l'aspect morphologique en L2 et des processus pouvant en rendre compte, à partir d'études que nous avons menées dans le cadre du programme européen sur l'acquisition de langues étrangères par des adultes: notamment sur l'acquisition non guidée du français L E par des hispanophones et des arabophones, du suédois par des hispanophones et des finnois. ${ }^{1}$ Ces études longitudinales sur des groupes d'apprenants ont permis d'établir des étapes acquisitionnelles qui sont franchies toujours dans le même ordre, et ce même pour des apprenants de couples de langues (L1 / L2) différents (cf. Dietrich, Klein \& Noyau 1995). Nous évoquerons aussi les résultats d'autres études longitudinales récentes recourant aux mêmes méthodologies et portant sur des apprenants guidés de divers groupes linguistiques, qui acquièrent la langue avec l'aide d'un dispositif d'enseignement. Et on constate, étonnamment peut-être, que les apprenants guidés, voire ayant comme seul environnement linguistique de L2 la salle de classe, manifestent eux aussi des étapes similaires - qu'ils dépassent généralement plus vite que les apprenants non guidés, heureusement. Mais le parcours d'acquisition (défini par les étapes successives de structuration) est très semblable dans ses principes chez tous les apprenants. Puis nous mettrons ces généralisations en rapport avec certaines positions avancées en typologie à propos des processus de grammaticalisation - plus spécifiquement de la morphologisation des verbes.

11 Notre approche fonctionnelle du développement de la L E chez les apprenants est fondée sur les principes suivants (cf. von Stutterheim \& Klein 1986, Trévise \& Porquier 1985, Noyau 1988) : les lectes des apprenants sont des constructions évolutives, dotées d'une structuration propre qui n'est ni celle de la L1 ni celle de la langue cible ; la structure d'un lecte d'apprenant peut être décrite par deux voies complémentaires: une voie onomasiologique (quel répertoire de moyens d'expression pour un champ notionnel, comme celui de la temporalité ?), et une voie sémasiologique (quelles valeurs ou 
fonctions ont les formes de ce lecte, par exemple un marqueur idiosyncrasique pré-verbal [le-] ?). A partir des résultats descriptifs obtenus par ces deux voies, les processus cognitifs de construction de la L E sont reconstruits de façon inductive, et leur généralisabilité est établie par comparaison entre apprenants individuels, entre groupes d'apprenants de différentes L E, ou de différentes L 1 acquérant une même L E, entre situations d'acquisition, en ayant soin de recourir aux mêmes méthodes d'investigation (obtention de données, analyses).

12 Aux premières étapes de la construction d'un lecte de LE, l'apprenant est confronté à un problème central, celui de la segmentation : comment arriver à segmenter l'input oral pour identifier des unités lexicales et des morphèmes? On sait par exemple que la morphologie orale du français est assez opaque, les liens formes-fonctions y sont difficiles à établir : formes plurifonctionnelles ( $\mathrm{V}-[\mathrm{e}] \mathrm{a} \mathrm{n}$ fonctions) ; flexions par affixation et par thèmes (cf. de Vogüé 1999) ; double fonction temporelle vs aspectuelle du PComp, etc.

13 Face à ce problème, les phases précoces d'expression de la temporalité chez les apprenants adultes de LE voient tout d'abord la mise en place d'un matériel lexical d'adverbes et expressions temporelles (von Stutterheim 1991, Noyau 1991, 1995, 1997, Starren 1996), qui peuvent devenir plus ou moins grammaticalisés alors que la morphologie verbale est encore absente (cf. Klein 1984, fertig V ( = V 'fini'), comme substitut du marquage de l'accompli). L'émergence de la morphologie temporelle tarde notablement sur le développement de la capacité à construire du discours ancré temporellement et spécifiant les relations temporelles entre événements, ce qui est fait par des moyens lexicaux, lexico-syntaxiques (connecteurs et subordonnants temporels), et par des organisations discursives correspondant à des configurations temporelles (principe de l'ordre chronologique pour les événements singuliers de la trame d'un récit, configuration cadre duratif - événement inclus). On a pu montrer que les lectes d'apprenants pouvaient se stabiliser à ce point, avant l'accès à la morphologie temporoaspectuelle (Klein 1 Perdue, 1997, Noyau 1991, 1998, sous presse).

question se pose alors : comment l'apprenant non guidé d'une langue orale en arrive-til à des hypothèses sur les variations de forme des lexèmes verbaux et sur leurs fonctions? Quelles fonctions temporelles trouvent en premier une expression grammaticalisée? Quels schèmes morphologiques sont construits pour constituer les premières oppositions morphologiques? Nous illustrerons ces points essentiellement à partir de l'acquisition du français par des adultes dans le milieu social, en rappelant que les études translinguistiques menées en parallèle dans plusieurs pays européens ont montré des convergences remarquables sur la structure de ces évolutions quel que soit le couple de langues (cf. Dietrich, Klein \& Noyau 1995).

Dans l'acquisition du français par des hispanophones, la première opposition devenant fonctionnelle - après une phase d'allomorphie verbale purement formelle - se fait entre les schèmes Aux-V-[e] V- $\varnothing$ ([travaj] [a travaje], [par(t) [aparti]), alors que le schème $\mathrm{V}$-[e] reste longtemps une forme neutre, temporellement non intégrée, apparaissant dans tous les contextes (Noyau 1990, 1991, 1998). Cette première opposition est clairement temporelle, et non pas aspectuelle, et l'opposition passé imperfectif / passé perfectif est acquise très tardivement (on retrouve ce résultat même chez les apprenants guidés de L1 romanes, cf. Garat 1996, Brum de Paula 1998).

16 En est-il de même pour des locuteurs dont la L1 est à dominante aspectuelle? Chez des apprenants arabophones de français dans le milieu social, on observe une longue période de profusion allomorphique des verbes (cf. Noyau 1998), avant que la place pré-verbale 
devienne le locus privilégié des tentatives de marquage ([par] / [epar], [lipar(ti)], [apar (ti)]), avec parfois des marqueurs idiosyncrasiques (cf. la tentative chez un locuteur de créer un schème [ma-]V-e (il [mareste], il [maparti]). Quelles hypothèses fonctionnelles sous-tendent ces tentatives de structuration? Un examen minutieux des alternances dans les productions successives d'un apprenant permet de conclure à une évolution de l'hypothèse nulle $\mathbf{H Ø ~ ( a l l o m o r p h i e ~ s a n s ~ f o n c t i o n ) ~ a ̀ ~ u n e ~ h y p o t h e ̀ s e ~ l e x i c a l e ~ f o n d e ́ e ~ s u r ~ l a ~}$ temporalité inhérente des procès $\mathrm{Hi}$ (procès téliques : schème $\mathrm{x}-\mathrm{V}$-[e] / procès duratifs atéliques : schème V-Ø), à une oscillation entre une hypothèse aspectuelle $\mathbf{H a}$ (perfectif / imperfectif ou bien simple / accompli - les contextes discursifs ne permettant généralement pas de trancher) et une hypothèse temporelle $\mathbf{H t}$ (révolu / actuel). A ce dernier stade $\mathbf{H a} / \mathbf{H t}$, certains types de procès sont plus susceptibles que d'autres de porter la marque du révolu ou de l'accompli : ceux qui s'associent de façon prototypique à l'achèvement. Les associations non-prototypiques entre procès duratif sans changement et marque d'accompli ou de révolu ( type [a dormi]) n'apparaissent que chez des apprenants beaucoup plus avancés, chez lesquels la grammaticalisation des tiroirs verbaux est solidement ancrée. ${ }^{2}$

Ches des apprenants plus avancés, on observe les étapes successives de la fonctionnalisation des oppositions morphologiques: quelles fonctions des marqueurs apparaissent en premier? L'étude des formes verbales à travers les récits successifs d'une Finnoise acquérant le suédois (Noyau 1991, 1995, 1997) montre que le prétérit se stabilise comme temps de base de la narration d'événements révolus du monde réel bien avant de marquer les verbes de la trame des récits de fiction, ce qui met bien en lumière la prépondérance de la fonction sémantique (temporelle) du prétérit : passé / non-passé, sur la fonction discursive: marquer les événements de la trame narrative. La fonction référentielle précède la fonction conventionnelle tenant à un type textuel.

Au total, sur quels principes repose le processus de la morphologisation acquisitionnelle? Aux phases précoces, l'acquisition procède par unités lexicalesisolées ([va] [ire] [ale]ou [syi] [ete] apparaissent tôt); puis on assiste à laformation de schèmes par ressemblance entre formes entrant de proche en proche en réseau (cf. Bybee 1991), l'acquisition morphologique est lente et graduelle, les schèmes se diffusant à de nouvelles bases lexicales, y compris avec construction de formes idiosyncrasiques ([rœesevu] pour 'reçu'). Les restructurations du système ne se manifestent pas comme la découverte et la mise en œuvre subite d'une règle permettant de passer d'une forme à une autre dérivée. Dans le débat entre conceptions de la morphologie (cf. Vandeloise 1990), l'étude de l'acquisition (Bybee 1991, Bybee \& Slobin 1982) conforte une conception lexicaliste de la morphologie prenant le mot comme unité de base et reposant sur l'analogie et les ressemblances de famille, contre une conception prenant le morphème comme unité de base et où les formes de surface sont dérivées par application de règles.

Les données de la morphologisation en L E permettent de reposer la question classique adressée à l'acquisition enfantine du langage : l'aspect est-il acquis avant le temps ? Cette question a fait l'objet de débats très vifs mais reposant en partie sur un malentendu (aspect vs temporalité inhérente des situations). Dans l'acquisition enfantine, il parait clair que les oppositions morphologiques précoces relèvent de l'aspect. De nombreux auteurs, dont Weist (1989), posent des tendances universelles dans le développement du temps linguistique chez l'enfant (cf. Schlyter 1990): les étapes successives de la construction d'une représentation linguistique de la temporalité seraient les suivantes : 
1) le système du moment de la parole ( $S, E, R$ indifférenciés dans les termes de Reichenbach) ;

2) le système du temps de l'événement : seul le moment de la situation peut précéder ou suivre MP, mais le moment en question reste fixé au moment de la parole (MQ = MP), et toutes situations sont envisagées de là soit comme accomplies laissant des traces, soit comme à venir (prospectif), soit comme en cours: il s'agit donc d'aspect, et non de temps ;

3) le système du temps de référence : les relations entre MQ et MS, et entre MQ et MP, sont libres, le système peut construire des notions aspectuelles mais aussi temporelles (MQ * MP).

Dans l'acquisition adulte de $\mathrm{L}$, on trouve l'inverse ( $\mathrm{MQ}=\mathrm{MS}$ et $\left.\mathrm{MQ}{ }^{*} \mathrm{MP}\right)$, donc la construction de notions purement temporelles, même si la L1 est à dominante aspectuelle.

21 C'est ce que montre l'acquisition tardive - et très lente - de l'Imparfait en français L2 (le schème V-[e]), ${ }^{4}$ de même qu'en italien ou en espagnol L2, où le tiroir verbal est pourtant bien identifiable, et l'acquisition tardive également du Parfait en suédois (sauf en tant que variante du prétérit, donc à valeur temporelle, comme en allemand). L'Imparfait est d'abord restreint à certains verbes - l'émergence des schèmes morphologiques est sensible aux caractéristiques temporelles inhérentes des procès: d'abord marqué seulement sur les auxiliaires, les verbes modaux, puis sur d'autres procès statiques, et beaucoup plus tardivement sur des procès transitionnels (cf. Andersen, Garat, Brum de Paula).

\section{Discussion}

Quels liens établir entre ces résultats et les études typologiques? Nous présentons quelques réflexions

A) sur les invariants de construction du temps et de l'aspect chez les apprenants dans le développement de la $\mathrm{L} \mathrm{E}$, en relation avec un modèle général de la morphologie flexionnelle ;

B) sur les différences éventuelles tenant aux L1 ou aux L2 ;

C) sur les parallèles qui peuvent être établis avec d'autres types de dynamique linguistique, comme la pidginisation, la créolisation.

\section{A. Invariants de construction du temps et de l'aspect chez les apprenants}

Bybee (1991) s'appuie sur les phénomènes acquisitionnels enfantins pour remettre en question la conception combinatoire et paradigmes-exceptions de la morphologie flexionnelle prenant le morphème comme unité de base, au profit d'une conception lexicaliste prenant le mot comme unité de base : 'les schèmes morphologiques émergent par comparaison de mots dans les paradigmes et entre ceux-ci'. ${ }^{5}$ Le lexique doit être vu non comme une liste d'entrées ou dictionnaire à seulement deux dimensions, mais 'plutôt comme un réseau hautement structuré et organisé où les entrées sont interreliées et entretiennent des relations de diverses sortes'. ${ }^{6}$ De plus, le lexique mental est dynamique et tous les mots n'ont pas le même statut: la force des représentations lexicales varie en 
fonction de l'usage ou du non-usage des unités lexicales; et tous les mots ne sont pas forcément stockés en mémoire, comme les mots complexes dérivables d'autres mots, surtout lorsqu'ils sont peu fréquents. Les données de l'acquisition de L2 vont tout à fait dans ce sens: la préférence pour certains tiroirs verbaux en fonction des types sémantiques de procès ; l'émergence des catégories de temps ou aspect se faisant d'abord sur des verbes irréguliers fréquents, dans l'acquisition de L1 comme dans celle de L2.

Bybee (1991), examinant les données issues de travaux acquisitionnels, propose un modèle de la morphologie flexionnelle lexicaliste, se construisant par réseaux de liens locaux entre formes, qui est à la fois un modèle de la langue et de l'acquisition. Pour chaque lexème, la forme de base est la plus fréquente, et la plus prototypique en usage : celle qui a la représentation la plus forte dans le lexique mental. L'acquisition est vue comme procédant par stockage d'unités lexicales, création de connexions par analogie entre unités, puis formation de schèmes (patterns) entre connexions, puis de règles passant d'une forme à une autre dérivée. Le caractère plus ou moins marqué d'une forme est local, il dépend de la force lexicale, du degré de relation (de pertinence) lexèmemarqueur, et des relations interparadigmes.

\section{B) sur les différences éventuelles tenant aux L1 ou aux L2}

Dans l'acquisition de l'italien L2, on constate une évolution plus rapide que dans celle du français L2, quoique selon les mêmes étapes. Dans une langue morphologiquement transparente, préservant sans amalgame les marques d'aspect, temps, mode, personne, les connexions entre formes débouchent plus rapidement sur des schèmes, qui se diffusent mieux. On peut également distinguer (contrairement à ce qui se passe pour le français, où le schème oral $\mathrm{V}$-[e] est ambigu) quel est le schème meilleur candidat pour la forme-de-base avant la temporalité morphologique : c'est l'infinitif V-re, et quel est le schème réalisant le plus précocément le marquage du passé : c'est le schème de participe passé V-to, sans auxiliaire.

Selon les langues, le caractère marqué / non-marqué formellement vs fonctionnellement des tiroirs verbaux a un effet sur le cours de l'acquisition. Ainsi, on peut opposer le français, où le Présent est du point de vue fonctionnel non-marqué temporellement, et du point de vue formel non-marqué aussi (le schème $V-\varnothing)$ ), et le suédois, où le Présent, qui du point de vue fonctionnel est non-marqué temporellement, est du point de vue formel une forme marquée (schème $\mathrm{V}$-[r]). Dans l'acquisition du français L2, le schème $\mathrm{V}$-[e] reste la forme neutre, non marquée en temps/aspect, pendant longtemps (schème ambigu), et le schème V-Ø s'oppose à un schème de non-présent $\mathrm{x}-\mathrm{V}-[\mathrm{e}] .^{7}$ Dans l'acquisition du suédois L2 à l'inverse, la relation de marquage est inverse au niveau fonctionnel et au niveau formel, elle est anti-iconique, ce qui provoque une difficulté pour les apprenants, ${ }^{8}$ qui gardent longtemps le schème V-[a] (de l'Infinitif, et dans certains contextes de Prétérit réduit) comme forme de base, non-marquée. Il se dégage alors une opposition initiale entre les schèmes V-[de/te] (du Prétérit) qui marque le passé, et V-[a] comme forme fonctionnellement neutre.

\section{C) Parallèles avec d'autres dynamiques linguistiques}

Myhill (1991) mène une discussion serrée sur les liens entre pidginisation, créolisation et acquisition. Contrairement aux positions connues de Bickerton, il montre, à partir de 
l'analyse des marqueurs temporo-aspectuels dans des corpus de discours en divers créoles, qu'on peut tracer un parallèle entre l'acquisition de langues étrangères et la pidginisation (mais pas la créolisation, qui est nativisation, extension des fonctions linguistiques, et donc des formes, lors de l'accession du parler au statut de langue première dans une communauté), et d'autre part la dé-créolisation, convergence vers une langue cible, pour ce qui est des phases plus avancées de l'acquisition.

Un lecte de langue étrangère est la base (pidgin à partir de quoi va se développer un créole), les traits des créoles qui sont similaires à des traits de lectes d'apprenants sont là via le pidgin qui leur a donné naissance. Les variétés les plus basilectales ont logiquement le moins de marquage temporo-aspectuel. On remarque également que l'émergence des catégories de temps ou aspect se fait d'abord sur des verbes irréguliers fréquents, dans l'acquisition de L1 et de L2, comme c'est le cas aussi dans l'évolution des créoles. Le domaine de la morphologisation temporo-aspectuelle montre que les processus d'acquisition de langues étrangères peuvent contribuer à la compréhension des processus de pidginisation.

\section{BIBLIOGRAPHIE}

ANDERSEN Roger (1991): Developmental sequences: the emergence of aspect marking in second language acquisition. In : T. HUEBNER \& C. A. FERGUSON, eds. Crosscurrents in second language acquisition and linguistic theories. Amsterdam, Benjamins, 305-324.

BATES, Elisabeth \& Brian MACWHINNEY, B. (1989) : Functionalism and the competition model. In The crosslinguistic study of sentence processing. .B. MacWhinney \& E. Bates (eds), Cambridge : Cambridge University Press, 3-73.

BRUM de PAULA, Mirian (1998) : L'appropriation de la temporalité morphologique en langue étrangère : contextes linguistiques d'émergence et de structuration. Thèse de doctorat, Université de Paris-X.

BYBEE Joan L. (1985) : Morphology. Amsterdam : Benjamins.

BYBEE Joan L. (1991) : Natural morphology : the organization of paradigms and language acquisition. In : T. HUEBNER \& C. A. FERGUSON, eds. Crosscurrents in second language acquisition and linguistic theories. Amsterdam, Benjamins, 67-92.

BYBEE Joan L. \& SLOBIN Dan I (1982) : Rules and schemas in the development and use of the English past tense. Language 58/2, 265-289.

DESCLÉS J.-Pierre \& Zlatka GUENTCHEVA (1997) : Grammaticalisation et typologie. Modèles Linguistiques 36 (XVIII/2) 'La grammaticalisation', 17-31.

DE VOGÜÉ, Sarah (1999) : Ni temps ni mode : le système flexionnel du verbe en français. In : Svetlana VOGELEER, Andrée BORILLO, Marcel vUILLAUME \& Carl VETTERS, eds. : La modalité sous tous ses aspects. Amsterdam / Atlanta : Rodopi, 'Cahiers Chronos' 4, pp. 93-114.+ 353 p. 
DIETRICH Rainer, KLEIN Wofgang \& Colette NOYAU (1995): The acquisition of temporality in a second language. Amsterdam/New York : Benjamins.

GARAT Josefina (1996) : L'acquisition de la temporalité par des apprenants en milieu institutionnel, futurs professeurs du F.L.E. : étude longitudinale. Thèse de doctorat, Université de Paris-X.

GIACALONE RAMAT Anna (1992) : Grammaticalization processes in the area of temporal and modal relations. Studies in Second Language Acquisition 14/3, 297-322.

GOSSELIN Laurent \& Jacques FRANÇoIS (1991) : Les typologies de procès : des verbes aux prédications. In : C. FUCHS, ed. Les typologies de procès. TraLiPhi XXIX, Paris Klincksieck.

HOUSEN Alex (1995) : It's about time. The acquisition of temporality in English-as-a-SecondLanguage in a multilingual educational context. Thèse de doctorat, Vrije Universiteit Brussel.

KAIL Michèle (1983) : L'acquisition du langage repensée : les recherches interlangues. I : Principales propositions théoriques. II : Spécificités méthodologiques et recherches empiriques. L'Année Psychologique 83, pp. 225-258 et pp. 561-596.

KAIL Michèle (1990) : Le traitement des données de langage : la prise des indices et leur utilisation. in D. Gaonac'h (ed.) Acquisition et utilisation d'une langue étrangère. l'approche cognitive. Le Français dans le Monde-Recherches \& Applications, $\mathrm{n}^{\circ}$ spécial, 70-80.

KAIL Michèle (1995) : Variations interlinguistiques et développement du langage. Revue de Phonétique Appliquée 112-113, 239-261.

KIHLSTEDT Maria (1998) : La référence au passé dans le dialogue. Etude de l'acquisition de la temporalité chez des apprenants dits avancés de français. PhD. Stockholm : Akademitryck.

KLEIN Wolfgang \& Clive PERDUE (1997) : The basic variety, or : could'nt natural languages be much simple ? Second language Research 13/4, The basic variety.

MYHILL John (1991) : Typological text analysis : tense and aspect in creoles and second languages. In : T. HUEBNER \& C. A. FERGUSON, eds. Crosscurrents in second language acquisition and linguistic theories. Amsterdam, Benjamins, 93-122.

NOYAU Colette (1988) : Recherches sur l'acquisition spontanée de langues étrangères dans le milieu social. Dialogues et Cultures (Québec), mai, p. 208-218.

NOYAU Colette (1990): Structure conceptuelle, mise en texte et acquisition d'une langue étrangère”. In J. François \& G. Denhière (éds.) : ‘Cognition et langage’ Langages 100, décembre, p. 101-114.

NOYAU Colette (1991) : Construction du récit, construction de la langue. La temporalité dans le discours narratif. Thèse pour le diplôme national d'habilitation à diriger des recherches, vol. 1 et 2 / 4 . Université de Paris VIII.

NOYAU Colette (1995): Chap. 6 'The acquisition of French', Chap. 7 'The acquisition of Swedish', de : Dietrich, Klein \& Noyau (eds.), pp. 145-260.

NOYAU Colette (1997) : Processus de grammaticalisation dans l'acquisition de langues étrangères : la morphologie temporelle. In : C. Martinot, ed. L'acquisition de la syntaxe en langue maternelle et en langue étrangère, Besançon, Annales Littéraires de l'Université de Franche-Comté n 631, p. 223-252. 
NOYAU Colette (1998) : Le développement de la temporalité dans le récit : interactions entre processus de construction des formes et processus de construction de textes. SILTA (Studi Italiani di Linguistica Teorica e Applicata) 1/1998, 111-132.

NOYAU Colette (sous presse) : Temporal relations in learner varieties : grammaticalization and discourse constructions. Chap. 4 de : Salaberry R. \& Y. Shirai (eds.) L2 acquisition of tense-aspect morphology. Amsterdam/New York : Benjamins.

SCHLYTER Suzanne (1990). The acquisition of French temporal morphemes in adults and in bilingual children. In : Bernini, G. \& Giacalone Ramat, A. (eds.) : La temporalità nell'acquisitione di lingue seconde. Milano : Franco Angeli.

STARREN Marianne (1996) : Temporal adverbials as a blocking factor in the grammaticalization process of L2 learners. In : T. van der AVOIRD \& M. CORSIUS, eds. Proceedings of the CLS Opening, Academic Year '96-'97, Tilburg, Center for Language Studies.

STUTTERHEIM Christiane von (1991): Narrative and description : temporal reference in second language acquisition. In : T. HUEBNER \& C. A. FERGUSON, eds. Crosscurrents in second language acquisition and linguistic theories. Amsterdam, Benjamins, 385-404.

STUTTERHEIM Christiane von \& KLEIN Wolfgang (1986) : A concept-oriented approach to second language studies. In : C. PFAFF, ed. First and second language acquisition processes. Cambridge : Newbury House, 191-205.

TERZAN-KOPECKY Carmen (1997) : Die Typologie der Abfolge von Spracherwerbsprozessen. Sprachtypologie und Universalienforschung 50, 348-357.

TRÉVISE Anne \& Rémy PORQUIER (1985) : Acquisition d'une langue 2 en milieu naturel : quelles méthodologies de description? Langue Française 68.

VANDELOISE Claude (1990) : Règles ou listes : l'arbitrage de la morphologie. Le Français Moderne 3/4, 224-234.

WEIST, Richard M. (1989) : Time concepts in language and thought : filling the Piagetian void from two to five years. Chap. 2 de : LEVI I. \& D. ZAKAY eds. Time and human cognition : a life span perspective.Amsterdam, Elsevier (North-Holland), 63-116.

\section{NOTES}

1. Pour plus de détails, se reporter à Noyau 1991, 1995, 1997, 1998, sous presse.

2. Ces associations préférentielles des marqueurs aspecto-temporels à des types de procès spécifiques sont caractéristiques également du processus de morphologisation lors de l'acquisition enfantine du langage.

3. $\mathrm{S}=$ speech time, $\mathrm{E}=$ event time, $\mathrm{R}=$ reference time, nous dirons moment de la parole $(\mathrm{MQ})$, moment de la situation (MSit), et moment en question (MQ).

4. Cf. Andersen 1991, Giacalone Ramat 1992, Noyau 1995, Garat 1996, Brum de Paula 1998.

5. 'morphological patterns emerge through the comparison of words within and across paradigms' (Bybee 1991: 68).

6. 'rather as a highly structured and organized network in which entries are interconnected and form relationships of various sorts' (Bybee 1991: 70).

7. où $x$ est un précurseur d'auxiliaire.

8. D'autant plus pour les apprenants dont la L1 est l'espagnol, qui sont face à une contradiction L1/L2, puisque le schème V-[r] est celui de l'infinitif en espagnol. 


\section{AUTEUR}

\section{COLETTE NOYAU}

Université de Paris-X-Nanterre. Equipe ‘Dynamiques des langues', UMR MoDyCo 7114 CNRS cnoyau@u-paris10.fr 